\title{
Correction to: Monoclonal Caveolin 1 Expression in the Differential Diagnosis of Malignant Pleural Mesothelioma and Pulmonary Adenocarcinoma: Is it Useful?
}

Zehra Bozdag $^{1} \cdot$ Ediz Tutar $^{1} \cdot$ Omer Faruk Dizibuyuk ${ }^{1} \cdot$ Kemal Bakir $^{1}$

Published online: 30 January 2020

(C) Arányi Lajos Foundation 2020

Correction to: Pathology \& Oncology Research https://doi.org/10.1007/s12253-019-00751-9

The original version of this article unfortunately contained an error in Fig. 1. Cav-1 expression in MPM and PA cases failed to show the histopathological details in Fig. 1 due to technical problem. The figure with the proper sharpness and clarity is shown in the next page.

The online version of the original article can be found at https://doi.org/ 10.1007/s12253-019-00751-9

Zehra Bozdag

zbozdagmd@gmail.com

1 Department of Pathology, Gaziantep University, Medical School,

Gaziantep, Turkey 


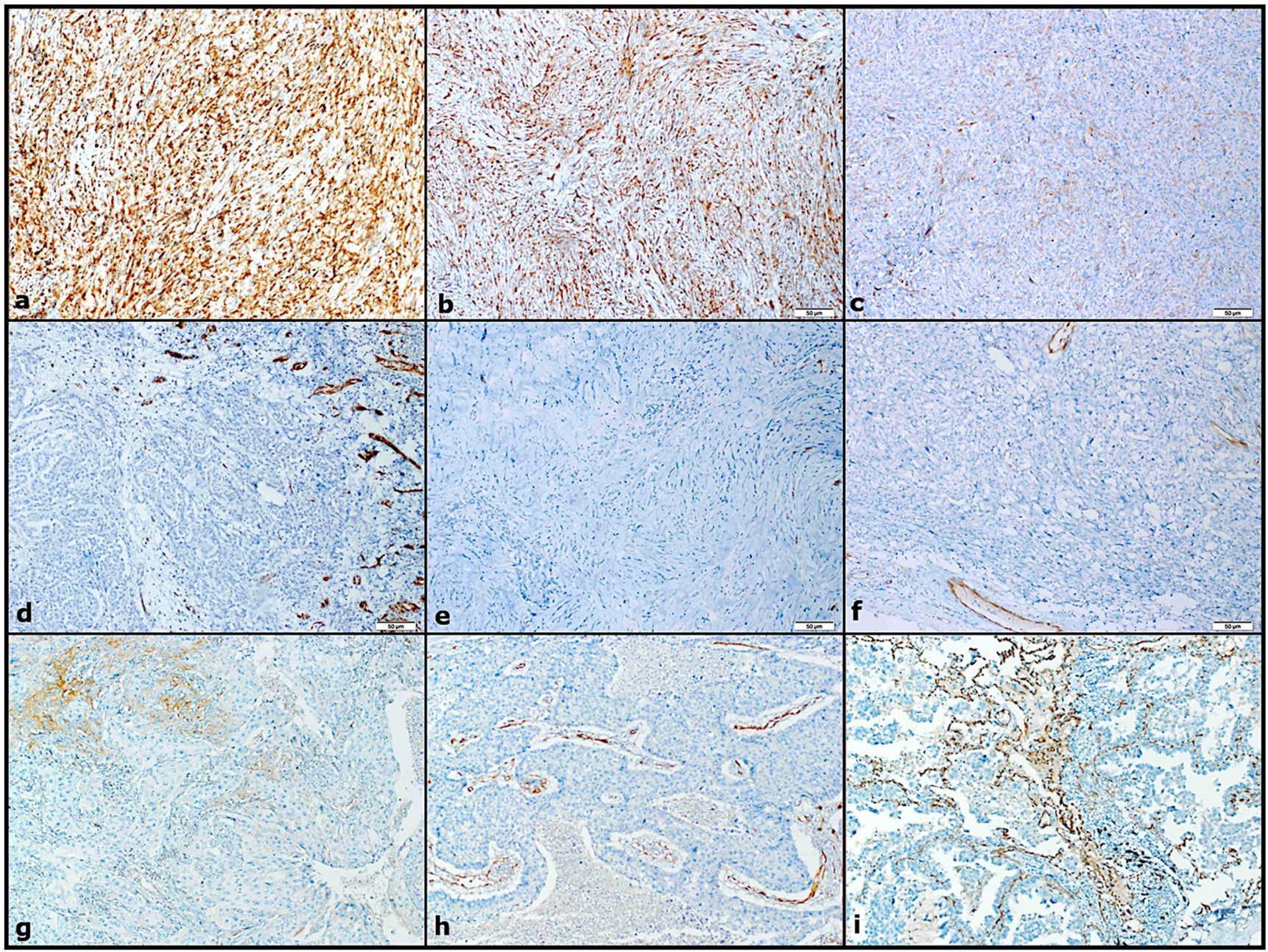

Fig. 1 Cav- 1 expression in MPM and PA cases; a: strong and score 3 (positivity in $>50 \%$ of tumor cells) expression in epitheloidmesothelioma $(\times 100)$, b: strong and score 3 expression in biphasic mesothelioma $(\times 100)$, c: weak and score 2 (positivity in $11-50 \%$ of tumor cells) expression in sarcomatoid mesothelioma $(\times 100)$, d: negative Cav-1 expression in epitheloid mesothelioma $(\times 100)$, e: negative Cav-1 expression in biphasic mesothelioma $(\times 100)$, f: negative Cav-1 expression in sarcomatoid mesothelioma $(\times 100)$, g:Moderate and score (1 positivity in $1-10 \%$ of tumor cells) expression in PA $(\times 100)$, h and i: negative Cav-1 expression in PA $(\times 100)$ 